\title{
Diffraction model of a plenoptic camera for in-situ space exploration
}

Ulrike Krutz, Martin Lingenauber, Klaus H. Strobl, Florian Fröhlich, Maximilian Buder

Ulrike Krutz, Martin Lingenauber, Klaus H. Strobl, Florian Fröhlich, Maximilian Buder, "Diffraction model of a plenoptic camera for in-situ space exploration," Proc. SPIE 10677, Unconventional Optical Imaging, 106772S (24 May 2018); doi: 10.1117/12.2306743

SPIE. Event: SPIE Photonics Europe, 2018, Strasbourg, France 


\title{
Diffraction model of a plenoptic camera for in-situ space exploration
}

\author{
Ulrike Krutz ${ }^{\mathrm{a}}$, Martin Lingenauber ${ }^{\mathrm{b}}$, Klaus H. Strobl ${ }^{\mathrm{b}}$, Florian Fröhlich ${ }^{\mathrm{b}}$, and Maximilian \\ Buder $^{\mathrm{a}}$ \\ ${ }^{a}$ DLR e.V., Institute of Optical Sensor Systems, Rutherfordstr. 2, Berlin, Germany \\ ${ }^{\mathrm{b}}$ DLR e.V., Institute of Robotics and Mechatronics, Münchener Str. 20, Weßling, Germany
}

\begin{abstract}
This paper provides a detailed system theoretical model of a plenoptic camera with the aim to provide in-depth understanding of the plenoptic data recording concept and its effects. Plenoptic cameras, also known as light field cameras, were firstly thought of in the beginning of the 20th century and became recently possible thanks to rapid development of processing hardware and the increase of camera sensor resolution. Despite being a new type of sensor, they are operated in the same way as conventional cameras, but offer several advantages. A plenoptic camera consists of a main lens and a lenslet array (microlens array) right in front of the detector. The microlens array causes not only the recording of the incident location of a light ray on the sensor, as it is done by a conventional camera, but also the incident direction. Such a record can be represented by a 4-D data set known as the light field. In fact, by inserting a microlens array any conventional camera can be transformed into a plenoptic camera. The plenoptic recording concept and the 4-D light field provide multiple advantages over conventional cameras. For example, a single recorded light field allows first, to reconstruct novel views with small changes in viewpoint, second, to create a depth map, and third, to refocus images after the data capture. Hence, the process of focusing is shifted from hardware to software. Last, but not least, plenoptic cameras allow an extended depth of field in comparison to a conventional camera and the use of a bigger camera aperture. Most of the mentioned advantages become particularly effective at close-range to an object. The German Aerospace Center performs research on plenoptic cameras for close-range imaging in space. Possible applications are for example robot vision with plenoptic cameras for robotic arm operations during on-orbit servicing missions or the use of plenoptic cameras on rovers in the course of exploration missions to other planets. Those application scenarios and the demanding conditions in space require thorough comprehension of plenoptic cameras. For this purpose, this paper shall provide a detailed model of plenoptic cameras, which allows to derive camera parameters and optimize them with particular attention to the user requirements and to generate synthetic data. The latter can be utilized to assess the evaluation algorithms, which are not mentioned in detail in this paper. The modeling of the plenoptic camera is mainly based on the theory of geometric optics expanded by elements of diffraction optics.
\end{abstract}

Keywords: plenoptic imaging, diffraction, space, stereo imaging

\section{INTRODUCTION}

During the last century, technology had to mature a lot before the idea of light field cameras, brought up by Frederic Ives and Gabriel Lippmann in 1903 and 1908, could flourish. The innovation was to make use of a lens array, thus creating multiple images on the detector. Each image now presented a slightly different perspective of the object making stereoscopic viewing possible. It is only now that it is possible to create precise microlens arrays, also called lenslet arrays. High-resolution cameras are available and advances in optical engineering and computational power enable us to evaluate digital photography in a way that was impossible to think of even a few decades ago. This development resulted in commercial, hand-sized plenoptic cameras developed by Lytro Inc. [1] and by Raytrix GmbH [2]. For a more detailed historical view of the plenoptic camera see [3].

Further author information: (Send correspondence to Ulrike Krutz)

Ulrike Krutz.: E-mail: ulrike.krutz@dlr.de, Telephone: +49 (0)3067055 526

Martin Lingenauber: E-mail: martin.lingenauber@dlr.de, Telephone: +49 (0)8153281595 
There are a couple of advantages over traditional imaging systems that underline the importance of ongoing investigations of light field cameras. For example, a single recorded light field allows the reconstruction of novel views with small changes in viewpoint [4]. Furthermore, a depth map can be created [5] and also images can be refocused after the data capture [6]. Hence, the process of focusing is shifted from hardware to software. Last, but not least, plenoptic cameras allow an Extended Depth Of Field (EDOF) in comparison to a conventional camera [7] and the use of a bigger camera aperture (i.e., to gather more light). Quite a convenient issue is that any conventional camera can be converted into a light field camera by inserting a microlens array (MLA) between lens and detector. During the last fifteen years the idea of light field cameras has been taken up again and it is subject to both research as well as to commercial activity. In research, the plenoptic camera might turn out to be a good alternative to stereo imaging as will be shown in the following sections.

After this short introduction we will first outline the two types of plenoptic cameras in section 2. In section 3 a possible space scenario for plenoptic cameras is introduced. Then, we will describe the plenoptic system in a geometrical way in section 4. A wave-optical description is given in section 5 , containing a diffraction model by Kirchhoff and Fresnel. We continue with a comparison between a plenoptic camera and a standard stereo camera in section 6 and close with a summary and an outlook in section 7 .

\section{PLENOPTIC IMAGING}

\subsection{Unfocused plenoptic camera}

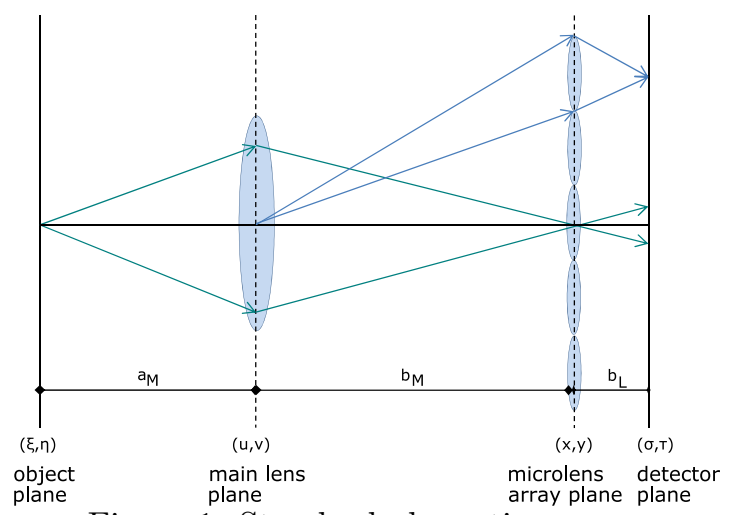

Figure 1: Standard plenoptic camera

In general, there exist two types of light field cameras. Based on Lippmann's idea [8], there is the so-called plenoptic camera I, which is the standard light field camera, or unfocused plenoptic camera. The term plenoptic (plenus is Latin for complete) is derived from the plenoptic function, first formulated by Adelson and Wang [9]. The plenoptic function describes each ray of light mathematically in terms of angular coordinates $(\Theta, \Phi)$, giving the direction of the ray, its wavelength $\lambda$, and the spatial coordinates $(x, y, z)$, representing the point this ray passes through, and time $t$ :

$$
P(\Theta, \Phi, \lambda, t, x, y, z) .
$$

Time can be neglected and light is monochromatic. Furthermore, in geometric optics, rays travel along straight lines with no change in radiance, making the z-coordinate irrelevant. Therefore (1) simplifies to a 4D function

$$
L(u, v, x, y)
$$

which is the so-called lumigraph [10]. Here, the angular coordinates $(\Theta, \Phi)$ yield to the more common Cartesian coordinates $(u, v)$. When we henceforth talk of the plenoptic function, we refer to (2).

The design of the unfocused plenoptic camera assumes the object to be imaged at the microlens array plane, while the microlenses focus at the main lens, see Figure 1. Furthermore, the main lens is considered to be at optical infinity. Let $a_{M}$ denote the distance between the object plane $(\xi, \eta)$ and the main lens plane $(\mathrm{u}, \mathrm{v}), b_{M}$ the distance between the main lens plane and the MLA plane $(\mathrm{x}, \mathrm{y})$, and, finally, $b_{L}$ the distance between the MLA plane and the detector plane $(\sigma, \tau)$. 


\subsection{Focused plenoptic camera}

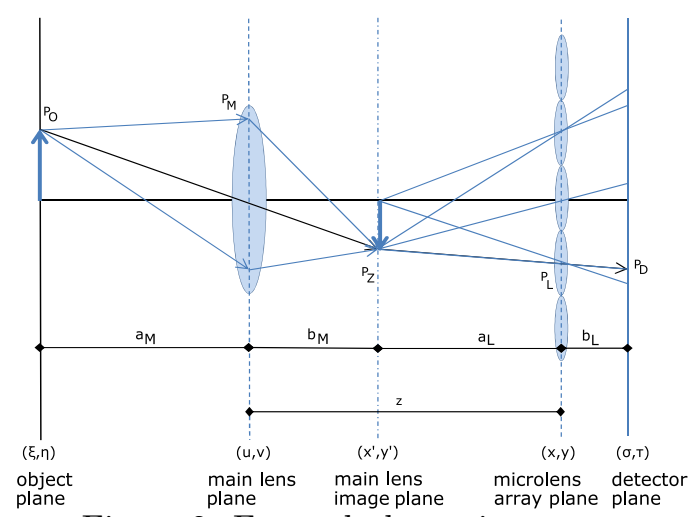

Figure 2: Focused plenoptic camera

The second type is the focused plenoptic camera, also known as plenoptic camera II. Here, the microlenses are not necessarily of the same type, but may differ in their placement and therefore also in their focal lengths. The main lens creates a virtual image of the object which lies in the main lens image plane between the main lens and the microlens array. Each microlens focuses on this virtual image and projects it to the detector plane, thus functioning as a relay system to the main lens [11], see Figure 2.

Here, $b_{M}$ denotes the distance between the main lens plane and the image plane of the main lens, $a_{L}$ the distance between the image plane of the main lens and the MLA plane, and $b_{L}$ the distance between the MLA plane and the detector plane, which is not necessarily where the microlenses are focused upon. The sum of $b_{M}$ and $a_{L}$ determines $z$, the distance between the main lens and the microlens array.

The so called multi-focus plenoptic camera is an improved version of the focused plenoptic camera [2,12]. Its MLA contains up to three different types of micro lenses, each with a different focal length, that are grouped together. The microlenses are designed such that their DOF just touch. By this, the DOF is extended even further than with a standard plenoptic camera. Once projected in object space, the DOF of a multi-focus plenoptic camera can be multiple times larger than the one of a conventional camera [2].

\section{PLENOPTIC CAMERAS FOR IN-SITU PLANETARY EXPLORATION}

In-situ planetary exploration means that measurements are conducted on the surface of a planet directly at a site of scientifc interest and not remotely from orbit. The necessary instruments are mounted on a lander or rover, where the latter offers the possibility to visit several sites of interest.

Amongst the many scientific instruments on board a rover, specialized cameras mounted on a robot arm are used to provide data of the smallest geological features such as grains of sand or fine cracks in rocks. These so called Hand Lens Imagers (HLI) are intended to work as a remotely operated version of the hand lens that a geologist uses during field trips for his investigations. As such they provide high resolution images that allow to determine the size of single grains and measure the length and width of cracks or of layers of a rock. From this information geologists can classify the materials and understand the geological history of a site in order to answer questions such as if there has been water or life on Mars [13]. Currently, the most prominent example for a HLI is the Mars Hand Lens Imager (MAHLI) on board the mars rover Curiosity [14]. HLIs are operated in close range to an object, i.e. with working distances as small as several centimeters in order to be able to achieve the required resolution.

When moved further away from an object, they provide images showing the context around a point of measurement or depending on their design they can also provide depth maps that were created from a sequence of overlapping images. Besides their scientific purpose, HLIs are also used for the operation and maintenance of the rover [15]. For this, they provide images and data that help to plan the operation of other instruments or to check the condition of the rover or parts of it. All those imaging tasks require well calibrated, radiometrically correct images that allow scientists, operators and engineers to get the same impression as if they were on the 
remote planet by themselves. Therefore in this context, cameras are more regarded to be measurement systems instead of simple imaging devices.

Due to their main purpose, imaging with HLIs is subject to the challenges and limitations of close range imaging. Most limiting is the shallow Depth of Field (DOF), which for example can be as small as $0.82 \mathrm{~mm}$ for a working distance of $22.5 \mathrm{~cm}$ for the MAHLI camera [16]. Therefore, MAHLI contains a focusable macro lens mechanism that allows to focus the camera from $22.5 \mathrm{~mm}$ to infinity [14]. The on-board image processing allows to combine up to 8 images, which were taken at different focus settings, to form a single EDOF image and to create an additional depth map by using a focus stacking algorithm [13].

But the use of mechanisms in space is prone to failure due to effects such as cold welding in vacuum, degradation caused by radiation or due to the fine and electrically charged dust on Moon or Mars. Thus they require sealed housings, special lubrication, careful design and extensive testing. Which, in the case of cameras, results in highly integrated and complex systems. This not only raises the size and mass of a camera system but also the risk of failure, not only during the operation but also already during the development [17].

On top of this, every system in space is accessible only via radio link, i.e. all mechanical, optical and electrical parts have to work flawless as no repair is possible. Plus, the signal runtime to remote planets often does not allow a direct control but requires some level of autonomy. All of this results in high demands on the reliability of each part. As a result, it means that avoiding mechanisms in space cameras, e.g. to change the focus or to change the aperture, can be highly beneficial, especially due the often small dimensions of such parts.

Thus it appears that plenoptic cameras are a promising concept for future HLIs due to the following reasons. First, the EDOF of plenoptic cameras makes it possible to omit a complex, space qualified focus mechanism that would be needed by a conventional camera in order to achieve the same DOF. And especially at close range, the DOF of a multi-focus plenoptic camera is multiple times larger when compared to the one of a conventional camera [2]. Second, by recording a light field, plenoptic cameras allow to create EDOF images, textured depth maps and novel views with small perspective changes from a single exposure. These are the data products geologists are expecting from a HLI for in-situ exploration. Additionally, when imaging objects further away or at infinity, plenoptic cameras provide conventional 2-D images which might show landscape views or alike. Hence, they can provide more data products than a conventional camera from a single exposure while being less complex.

A rover during an exploration mission usually follows a one-way trajectory and it stops from time to time at a site of scientific interest to perform a multitude of measurements. Because each site is visited only once, also each measurement performed there is a one time opportunity with no chance of repetition in order to correct mistakes. Additionally, each measurement is the base for the work of multiple scientists and can therefore affect the scientific results in many ways. Hence, measurements during planetary exploration have to be as correct as possible and require reliable and well calibrated instruments whose limits and performance must be known in detail.

Because plenoptic cameras are computational photography devices [18], the processing of the recorded light field is an important part of the imaging process. But the light field processing depends on assumptions about the optical system, thus its exact modeling will help to create more precise and reliable algorithms and as a result better data for the scientists. Therefore, one of the first steps towards plenoptic HLIs for in-situ exploration is the full understanding of the image formation process and the models presented in the following sections shall provide the necessary insight.

\section{GEOMETRIC DESCRIPTION OF A PLENOPTIC SYSTEM}

To get a first order estimation of the optical system of a light field camera, it is convenient to consider ray tracing. This approach gives information about the footprints that a light ray makes by passing the system but without taking diffraction or aberration into account. Consider the system given in Figure 2. We have a main lens plane $(\mathrm{u}, \mathrm{v})$, a microlens array plane $(\mathrm{x}, \mathrm{y})$, and a detector plane $(\sigma, \tau)$. The focal lengths $f_{M}$ of the main lens and $f_{L}$ of the microlenses are arbitrary, even though they should be chosen dependent from each other due to the f-number matching rule. This rule states that the f-number of the microlens array should be matched to the image-side f-number of the objective lens to guarantee the exhaustion of the full resolution of the image sensor 
[19]. We assume $z>>b_{L}$. The object plane is given by $(\xi, \eta)$. In addition, $P_{O}$ is a point in the object plane, $P_{Z}$ a point in the virtual plane $\left(x^{\prime}, y^{\prime}\right)$, and $P_{D}$ the point in the detector plane to which the microlens projects $P_{Z}$ from the virtual image plane.

\subsection{Mapping}

\subsubsection{Projection from object plane to detector plane: $P_{O} \rightarrow P_{D}$}

Let the center of the main lens plane be the origin of the coordinate system. $P_{O}\left(\xi, \eta,-a_{M}\right)$ shall be a certain point in the object plane. The aim of this calculation is to find out where at the detector plane the light emerging $P_{O}$ causes the image point $P_{D}$. Due to lens equation and image equations it is $b_{M}=a_{M} f_{M} /\left(a_{M}-f_{M}\right)$ and $x^{\prime}=\xi f_{M} /\left(f_{M}-a_{M}\right)$, resp. $y^{\prime}=\eta f_{M} /\left(f_{M}-a_{M}\right)$. First, $P_{O}$ is projected to $P_{Z}$ in the virtual image plane in the following way:

$$
P_{O}\left(\xi, \eta,-a_{M}\right) \longrightarrow P_{Z}\left(x^{\prime}, y^{\prime}, b_{M}\right)=P_{Z}\left(\frac{\xi f_{M}}{f_{M}-a_{M}}, \frac{\eta f_{M}}{f_{M}-a_{M}}, \frac{a_{M} f_{M}}{a_{M}-f_{M}}\right) .
$$

Now we consider the point $P_{D}^{i}$, where the ray hits the detector after passing a certain microlens $i$ with $x_{o f f}^{i}$ and $y_{o f f}^{i}$ as its center. Due to the image equations it is

$$
\sigma^{i}=-\frac{\xi f_{M} b_{L}}{a_{M} f_{M}-z\left(a_{M}-f_{M}\right)}+\frac{x_{o f f}^{i}\left(f_{M}-a_{M}\right) b_{L}}{a_{M} f_{M}-z\left(a_{M}-f_{M}\right)}
$$

resp.,

$$
\tau^{i}=-\frac{\eta f_{M} b_{L}}{a_{M} f_{M}-z\left(a_{M}-f_{M}\right)}+\frac{y_{o f f}^{i}\left(f_{M}-a_{M}\right) b_{L}}{a_{M} f_{M}-z\left(a_{M}-f_{M}\right)} .
$$

Therefore it is

$$
\begin{aligned}
& P_{D}^{i}\left(\sigma^{i}, \tau^{i}, b_{M}+b_{L}+a_{L}\right) \\
& =\left(-\frac{\xi f_{M} b_{L}}{a_{M} f_{M}-z\left(a_{M}-f_{M}\right)}+\frac{x_{o f f}^{i}\left(f_{M}-a_{M}\right) b_{L}}{a_{M} f_{M}-z\left(a_{M}-f_{M}\right)},-\frac{\eta f_{M} b_{L}}{a_{M} f_{M}-z\left(a_{M}-f_{M}\right)}+\frac{y_{o f f}^{i}\left(f_{M}-a_{M}\right) b_{L}}{a_{M} f_{M}-z\left(a_{M}-f_{M}\right)}, b_{M}+b_{L}+a_{L}\right) .
\end{aligned}
$$

\subsubsection{Projection from detector plane to object plane: $P_{D} \rightarrow P_{O}$}

Let now $P_{D}$ be a certain point on the detector. The following equation determines the point $P_{O}$ in the object plane which caused $P_{D}$. Converting equation (4) to $\xi$ and equation (5) to $\eta$ yields to

$$
P_{O}\left(\xi, \eta,-a_{M}\right)=\left(x_{o f f}^{i}-\frac{\sigma^{i} z}{b_{L}}+a_{M}\left(\frac{\sigma^{i} z}{f_{M} b_{L}}-\frac{\sigma^{i}}{b_{L}}-\frac{x_{o f f}^{i}}{f_{M}}\right), y_{o f f}^{i}-\frac{\tau^{i} z}{b_{L}}+a_{M}\left(\frac{\tau^{i} z}{f_{M} b_{L}}-\frac{\tau^{i}}{b_{L}}-\frac{y_{o f f}^{i}}{f_{M}}\right),-a_{M}\right) .
$$

\subsection{Deduction of the distance $a_{M}$ for two given image points of the same object}

If the distance between object and main lens is not known, the only hint is, that all points which could cause $P_{D}$ are on one straight line: $\xi=c_{1}+a_{M} c_{2}$. Let $P_{D}$ be a point in the detector plane, that appears in (at least) two adjacent microlens images with coordinates $\sigma^{1}$ and $\sigma^{2}$ according to the microlenses $i=1$ and $j=2$. From the knowledge of the coordinates and the offset it is possible to deduce the distance $a_{M}$ the original point has to the main lens plane, as the two straight lines will intersect at the coordinates of the original image point at that certain distance:

$$
a_{M}=f_{M}\left[1+\frac{f_{M}}{\left(z-f_{M}\right)-b_{L} \frac{x_{o f f}^{1}-x_{o f f}^{2}}{\sigma^{1}-\sigma^{2}}}\right] .
$$



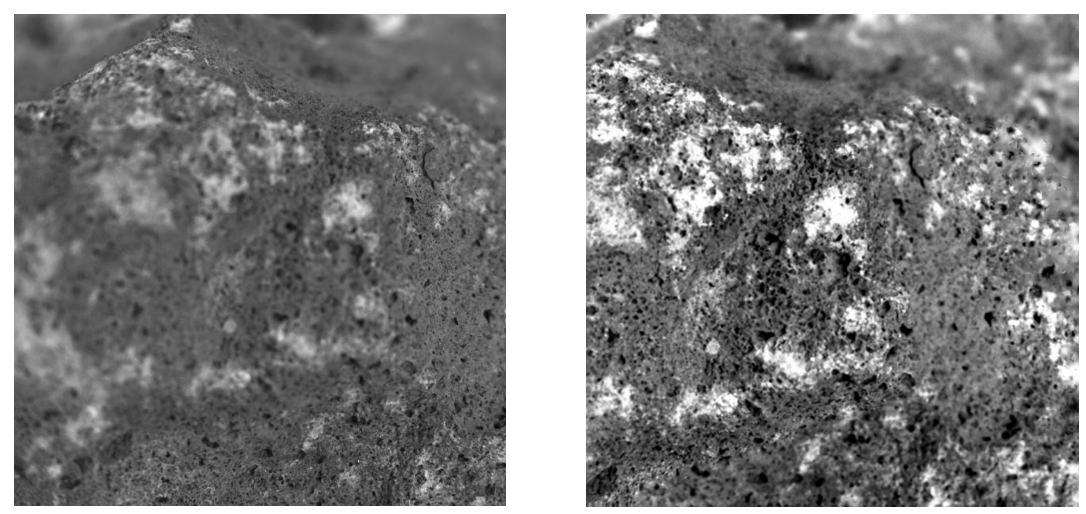

Figure 3: Example of an in-situ scene with a recorded rock surface with some depth extend. Left: conventional camera image. Right: multi-focus plenoptic camera image with an extended depth of field.

\subsection{Geometric model for space scenario}

As mentioned in section 3 plenoptic cameras might find applications during in-situ planetary exploration missions. Figure 3 shows such a conceivable scenario of an in-situ mission. The left image shows a scene of a rocky area recorded by a conventional camera at a distance of approximately $160 \mathrm{~mm}$. Due to the camera's shallow DOF only some parts of the rock are in focus and the rest appears blurry. The right image shows the same scene captured by a plenoptic color camera (Raytrix R5-C) at a distance of $140 \mathrm{~mm}$ with an EDOF due to the light field processing.
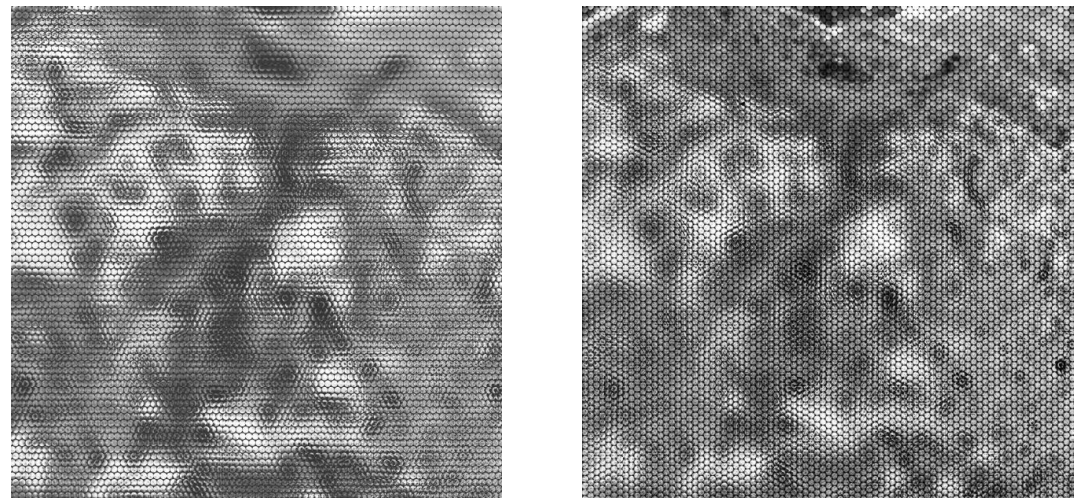

Figure 4: Comparison of a real plenoptic raw image with a simulate raw image. Left: Simulated raw plenoptic image with depth. Right: Real raw plenoptic image.

Figure 4 on the right shows the raw image from a real plenoptic camera which was used to derive the EDOF image shown on the right in Figure 3. It has been de-Bayered, histogram-equalized, and grey-scaled. The left image in Figure 4 shows a simulated raw image made with the proposed geometric model. Since the input image used for the simulation is only 2-dimensional, the resulting image is lacking in depth. The Raytrix raw image, a real test image of a 3-D object, includes depth information. The difference can be clearly seen in the upper part of the left and the right image of Figure 4.

\section{WAVE-OPTICAL DESCRIPTION OF A PLENOPTIC SYSTEM}

Camera systems consist of two components: the optics and the detector. While the optics capture and focus the light, the detector converts the striking photons into electrons. The amount of electrons can be assigned to a digital number via an analog-digital-converter. This number corresponds to a certain brightness value in 
the picture. Furthermore, there are camera parameter describing the optical system such as the Point Spread Function (PSF), which has an effect on the resolution performance of the camera. This effect is responsible for to what extent an object can be reproduced by a sensor system [20].

Nowadays, the pixel sizes of detectors usually range in the order of some visible light wavelengths. Combined with a low aperture, effects of diffraction can be observed and a wave-theoretical approach is convenient. The PSF of the optics can be determined using several methods. There are a few options in practice like measuring the PSF by depressing known spatial signals or determining the optical transfer function (OTF), which is the Fourier transform of the PSF, from the auto correlation signal of the pupil function. Another possibility is to get the modulation transfer function (MTF) from the Wiener spectrum of a noise distribution in the image plane [21]. Here, however, a theoretical approach is used. There are three possibilities of mathematical determination. Either it can be calculated by using the diffraction integral of Kirchhoff, or the equations by Fresnel or Fraunhofer. Kirchhoff is the base for Fresnel, which gives a quadratic approximation of Kirchhoff, as well as for Fraunhofer, which simplifies Kirchhoff to a linear function. The equation by Kirchhoff underlies so-called boundary conditions. It is assumed that the output amplitude of the wave of the object to be mapped turns rapidly to zero at the edge of the aperture. In reality, this is usually not correct. Furthermore, the polarization of light is neglected. Instead of using vectors, scalars are used for amplitude calculations. Therefore, Kirchhoff itself is only an approximation [22]. The approximation by Fresnel offers quite a few advantages concerning calculations for camera systems. For example, modeling the diffraction effects can be simplified by replacing the square root in the phase term by a quadratic form, by using other approximations [23]. The Fraunhofer approximation is not relevant for this work as it is only valid for far-field observations.

Up to now, there are only a few works dealing with a wave-optical approach for plenoptic cameras, like [24] or [25]. However, these publications concentrate on the Fresnel approximation, which is not always valid [26]. The calculations in this paper focus on Kirchhoff, as well as on Fresnel.

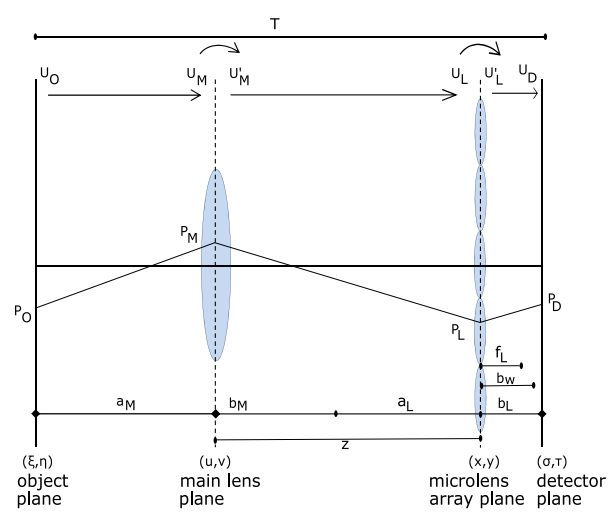

Figure 5: Calculation of amplitudes

Consider the system given in Figure 5. This system resembles any plenoptic camera (focused or unfocused) as there is a main lens plane $(\mathrm{u}, \mathrm{v})$, a MLA plane $(\mathrm{x}, \mathrm{y})$ and a detector plane $(\sigma, \tau)$. The focal lengths $f_{M}$ and $f_{L}$ are here arbitrary, though they should be chosen dependent from each other according to the f-number matching rule. Furthermore, it is $z=b_{M}+a_{L}$ the distance between main lens plane and MLA plane, while $b_{M}$ denotes the focal length of the main lens, which might reach the microlens array plane, as it is meant for the plenoptic camera I. The derived equations allow this. All variables are the same as described in subsection 2.2. Additionally, $U_{O}$ is the amplitude right behind the object plane, $U_{M}$ the amplitude right in front of the main lens plane, $U_{M}^{\prime}$ the amplitude directly behind the main lens, $U_{L}$ the amplitude in front of the MLA plane, $U_{L}^{\prime}$ the amplitude right behind the MLA, and, finally, $U_{D}$ the amplitude directly in front of the detector plane.

\subsection{PSF by Kirchhoff}

Let $\overrightarrow{r_{O}}$ be the vector $\overrightarrow{P_{O} P_{M}}, \overrightarrow{r_{M}}$ the vector $\overrightarrow{P_{M} P_{L}}$ and $\overrightarrow{r_{L}}$ the vector $\overrightarrow{P_{L} P_{D}}$. Furthermore, $\overrightarrow{n_{O}}, \overrightarrow{n_{M}}$ and $\overrightarrow{n_{L}}$ denote the normal vectors emerging $P_{O}, P_{M}$ and $P_{L}$. Besides, $A_{M}$ depicts the geometry of the main lens, in 
this case a circle. $R_{M}$ is the radius of the aperture of the main lens. Taking the lens into account, the phase $\varphi_{\lambda}^{M}(u, v)=\frac{2 \pi}{\lambda} n_{\lambda}^{M} \Delta_{0}^{M}-\frac{\pi}{\lambda f_{M}}\left(u^{2}+v^{2}\right)$ must be included to the equations beneath. Here, $f_{M}$ is the focal length of the main lens, $n_{\lambda}^{M}$ the refractive index and $\Delta_{0}^{M}$ the maximum thickness of the main lens. With this, the phase transition is given by $\Phi_{\lambda}^{M}(u, v)=A^{M}(u, v) e^{i \varphi_{\lambda}^{M}(u, v)}$. Likewise, the equations for the microlens can be derived. With the diffraction formula by Kirchhoff, the amplitude right in front of the main lens is calculated by

$$
U_{\lambda}^{M}\left(P_{M}\right)=\iint_{O} d O \frac{e^{\frac{2 \pi i}{\lambda}\left|\overrightarrow{r_{O}}\right|}}{i \lambda\left|\overrightarrow{r_{O}}\right|} \cdot \cos \left(\overrightarrow{n_{O}}, \overrightarrow{r_{O}}\right) \cdot U_{\lambda}^{O}\left(P_{O}\right)
$$

This can be transformed into

$$
U_{\lambda}^{M}(u, v)=\frac{a_{M}}{i \lambda} \cdot \iint d \xi d \eta \frac{e^{\frac{2 \pi i}{\lambda}\left|\overrightarrow{r_{O}}\right|}}{\left|\overrightarrow{r_{O}}\right|^{2}} \cdot U_{\lambda}^{O}(\xi, \eta)
$$

Using the phase transition, the amplitude right behind the main lens is given by

$$
U_{\lambda}^{\prime}{ }^{M}(u, v)=\frac{a_{M}}{i \lambda} \cdot e^{\frac{2 \pi i}{\lambda} n_{M} \Delta_{M}} \cdot e^{-\frac{\pi i}{\lambda f_{M}}\left(u^{2}+v^{2}\right)} \iint d \xi d \eta \frac{e^{\frac{2 \pi i}{\lambda}\left|\overrightarrow{r_{O}}\right|}}{\left|\overrightarrow{r_{O}}\right|^{2}} \cdot U_{\lambda}^{O}(\xi, \eta)
$$

Now the propagation from main lens to the lenslet array must be taken into account. It is considered $x^{\prime}:=x-x_{0}$ and $y^{\prime}:=y-y_{0}$, so it is not necessarily a point on the optical axis. Then,

$$
U_{\lambda}^{L}\left(x^{\prime}, y^{\prime}\right)=\frac{a_{M} \cdot z}{-\lambda^{2}} \cdot e^{\frac{2 \pi i}{\lambda} n_{M} \Delta_{M}} \iint d u d v e^{-\frac{\pi i}{\lambda f_{M}}\left(u^{2}+v^{2}\right)} \iint d \xi d \eta \frac{e^{\frac{2 \pi i}{\lambda}\left(\left|\overrightarrow{r_{M}}\right|+\left|\overrightarrow{r_{O}}\right|\right)}}{\left|\overrightarrow{r_{M}}\right|^{2}\left|\overrightarrow{r_{O}}\right|^{2}} \cdot U_{\lambda}^{O}(\xi, \eta),
$$

and, after the phase shift from the microlens,

$$
\begin{aligned}
U_{\lambda}^{\prime}{ }_{\lambda}^{L}\left(x^{\prime}, y^{\prime}\right)= & \frac{a_{M} \cdot z}{-\lambda^{2}} \cdot e^{\frac{2 \pi i}{\lambda}\left(n_{M} \Delta_{M}+n_{L} \Delta_{L}\right)} \cdot e^{-\frac{\pi i}{\lambda f_{L}}\left(x^{\prime 2}+y^{\prime 2}\right)} \\
& \times \iint d u d v e^{-\frac{\pi i}{\lambda f_{M}}\left(u^{2}+v^{2}\right)} \iint d \xi d \eta \frac{e^{\frac{2 \pi i}{\lambda}\left(\left|\overrightarrow{r_{O}}\right|+\left|\overrightarrow{r_{M}}\right|\right)}}{\left|\overrightarrow{r_{O}}\right|^{2}\left|\overrightarrow{r_{M}}\right|^{2}} \cdot U_{\lambda}^{O}(\xi, \eta) .
\end{aligned}
$$

The amplitude right in front of the detector plane, collecting light from all microlenses $(\# M L)$, is

$$
\begin{aligned}
U_{\lambda}^{D}\left(\sigma^{\prime}, \tau^{\prime}\right)= & \frac{a_{M} \cdot z \cdot b_{L}}{-i \lambda^{3}} \cdot e^{\frac{2 \pi i}{\lambda}\left(n_{M} \Delta_{M}+n_{L} \Delta_{L}\right)} \\
& \times \sum_{k, l=0}^{\# M L-1} \iint d x_{k}^{\prime} d y_{l}^{\prime} e^{-\frac{\pi i}{\lambda f_{L}}\left(x_{k}^{\prime 2}+y_{l}^{\prime 2}\right)} \iint d u d v e^{-\frac{\pi i}{\lambda f_{M}}\left(u^{2}+v^{2}\right)} \\
& \times \iint d \xi d \eta \frac{e^{\frac{2 \pi i}{\lambda}\left(\left|\overrightarrow{r_{O}}\right|+\left|\overrightarrow{r_{M}}\right|+\left|\overrightarrow{r_{L}}\right|\right)}}{\left|\overrightarrow{r_{O}}\right|^{2}\left|\overrightarrow{r_{M}}\right|^{2}\left|\overrightarrow{r_{L}}\right|^{2}} \cdot U_{\lambda}^{O}(\xi, \eta) .
\end{aligned}
$$

It is

$$
U_{\lambda}^{D}\left(\sigma^{\prime}, \tau^{\prime}\right)=\iint d \xi d \eta h_{\lambda}\left(\xi, \eta, \sigma^{\prime}, \tau^{\prime}\right) U_{\lambda}^{O}(\xi, \eta)
$$

The PSF results into

$$
\begin{aligned}
h_{\lambda}\left(\xi, \eta, \sigma^{\prime}, \tau^{\prime}\right)= & \frac{a_{M} \cdot z \cdot b_{L}}{-i \lambda^{3}} \cdot e^{\frac{2 \pi i}{\lambda}\left(n_{M} \Delta_{M}+n_{L} \Delta_{L}\right)} \\
& \times \sum_{k, l=0}^{\# M L-1} \iint d x_{k}^{\prime} d y_{l}^{\prime} e^{-\frac{\pi i}{\lambda f_{L}}\left(x_{k}^{\prime 2}+y_{l}^{\prime 2}\right)} \iint d u d v e^{-\frac{\pi i}{\lambda f_{M}}\left(u^{2}+v^{2}\right)} \frac{e^{\frac{2 \pi i}{\lambda}\left(\left|\overrightarrow{r_{O}}\right|+\left|\overrightarrow{r_{M}}\right|+\left|\overrightarrow{r_{L}}\right|\right)}}{\left|\overrightarrow{r_{O}}\right|^{2}\left|\overrightarrow{r_{M}}\right|^{2}\left|\overrightarrow{r_{L}}\right|^{2}} .
\end{aligned}
$$




\subsection{PSF by Fresnel}

Equation (9) is the starting point for the approximation by Fresnel. Fresnel's considerations are based on a remote field observation, i.e., it is assumed that $|\xi|,|\eta| \ll a_{M}$ and $|u|,|v| \ll a_{M}$. Furthermore the angular between $\overrightarrow{r_{O}}$ and $\overrightarrow{n_{O}}$ is considered so small, that cosine can be set to 1 . Another consequence resulting from far field observation is to regard $\left|\overrightarrow{r_{O}}\right|$ in the denominator as a constant and equal to $a_{M}$. The term in the exponent, containing the essential phase information for the superposition of the waves, must now be considered as a constant and is approximated by Taylor therefore:

$$
\left|\overrightarrow{r_{O}}\right| \approx a_{M} \cdot\left[1+\frac{1}{2} \frac{(u-\xi)^{2}}{a_{M}^{2}}+\frac{1}{2} \frac{(v-\eta)^{2}}{a_{M}^{2}}\right] .
$$

Likewise $\left|\overrightarrow{r_{M}}\right|$ and $\left|\overrightarrow{r_{L}}\right|$ are approximated. By this, the spherical waves are replaced by parabolic wave fronts [27]. Now, the amplitude in front of the main lens is given by

$$
U_{\lambda}^{M}(u, v)=\frac{e^{\frac{2 \pi i}{\lambda} a_{M}}}{i \lambda a_{M}} \iint d \xi d \eta e^{\frac{\pi i}{\lambda a_{M}}\left[(u-\xi)^{2}+(v-\eta)^{2}\right]} U_{\lambda}^{O}(\xi, \eta) .
$$

The amplitude behind the main lens is

$$
U_{\lambda}^{\prime}{ }^{M}(u, v)=\frac{e^{\frac{2 \pi i}{\lambda} a_{M}}}{i \lambda a_{M}} e^{\frac{2 \pi i}{\lambda} n_{M} \Delta_{M}} \cdot e^{-\frac{\pi i}{\lambda f M}\left(u^{2}+v^{2}\right)} \iint d \xi d \eta e^{\frac{\pi i}{a_{M}}\left[(u-\xi)^{2}+(v-\eta)^{2}\right]} U_{\lambda}^{O}(\xi, \eta) .
$$

In the same way, the amplitude in front of a microlens is calculated by

$$
\begin{aligned}
U_{\lambda}^{L}\left(x^{\prime}, y^{\prime}\right)= & \frac{e^{\frac{2 \pi i}{\lambda}\left(a_{M}+z\right)}}{-\lambda^{2} a_{M} \cdot z} \cdot e^{\frac{2 \pi i}{\lambda} n_{M} \Delta_{M}} \\
& \times \iint d u d v e^{-\frac{\pi i}{\lambda f}\left(u^{2}+v^{2}\right)} \\
& \times \iint d \xi d \eta e^{\frac{\pi i}{\lambda a_{M}}\left[(u-\xi)^{2}+(v-\eta)^{2}\right]} e^{\frac{\pi i}{\lambda z}\left[\left(x^{\prime}-u\right)^{2}+\left(y^{\prime}-v\right)^{2}\right]} U_{\lambda}^{O}(\xi, \eta)
\end{aligned}
$$

and, with the phase shift, the amplitude behind the microlens results in

$$
\begin{aligned}
U_{\lambda}^{\prime}{ }_{\lambda}^{L}\left(x^{\prime}, y^{\prime}\right)= & \frac{e^{\frac{2 \pi i}{\lambda}\left(a_{M}+z\right)}}{-\lambda^{2} a_{M} \cdot z} \cdot e^{\frac{2 \pi i}{\lambda}\left(n_{M} \Delta_{M}+n_{L} \Delta_{L}\right)} e^{-\frac{\pi i}{\lambda f_{L}}\left(x^{\prime 2}+y^{\prime 2}\right)} \\
& \times \iint d u d v e^{-\frac{\pi i}{\lambda f_{M}}\left(u^{2}+v^{2}\right)} \\
& \times \iint d \xi d \eta e^{\frac{\pi i}{\lambda a_{M}}\left[(u-\xi)^{2}+(v-\eta)^{2}\right]} e^{\frac{\pi i}{\lambda z}\left[\left(x^{\prime}-u\right)^{2}+\left(y^{\prime}-v\right)^{2}\right]} U_{\lambda}^{O}(\xi, \eta) .
\end{aligned}
$$

Finally, the amplitude in front of the detector plane $\left(\sigma^{\prime}:=\sigma-\sigma_{0}, \tau^{\prime}:=\tau-\tau_{0}\right)$ can be determined:

$$
\begin{aligned}
U_{\lambda}^{D}\left(\sigma^{\prime}, \tau^{\prime}\right)= & \frac{e^{\frac{2 \pi i}{\lambda}\left(a_{M}+z+b_{L}\right)}}{-i \lambda^{3} a_{M} \cdot z \cdot b_{L}} \cdot e^{\frac{2 \pi i}{\lambda}\left(n_{M} \Delta_{M}+n_{L} \Delta_{L}\right)} \\
& \times \sum_{k, l=0}^{\# M L-1} \iint d x_{k}^{\prime} d y_{l}^{\prime} e^{-\frac{\pi i}{\lambda f_{L}}\left(x_{k}^{\prime 2}+y_{l}^{\prime 2}\right)} e^{\frac{\pi i}{\lambda b_{L}}\left[\left(\sigma-x_{k}^{\prime}\right)^{2}+\left(\tau-y_{l}^{\prime}\right)^{2}\right]} \\
& \times \iint d u d v e^{-\frac{\pi i}{\lambda f_{M}}\left(u^{2}+v^{2}\right)} e^{\frac{\pi i}{\lambda z}\left[\left(x_{k}^{\prime}-u\right)^{2}+\left(y_{l}^{\prime}-v\right)^{2}\right]} \\
& \times \iint d \xi d \eta e^{\frac{\pi i}{\lambda a_{M}}\left[(u-\xi)^{2}+(v-\eta)^{2}\right]} .
\end{aligned}
$$


By sorting terms differently the following equation is obtained:

$$
\begin{aligned}
U_{\lambda}^{D}\left(\sigma^{\prime}, \tau^{\prime}\right)= & -\frac{e^{\frac{2 \pi i}{\lambda}\left(a_{M}+z+b_{L}\right)}}{i \lambda^{3} \cdot a_{M} \cdot z \cdot b_{L}} \cdot e^{\frac{\pi i}{\lambda b_{L}}\left(\sigma^{\prime 2}+\tau^{\prime 2}\right)} \cdot e^{\frac{2 \pi i}{\lambda}\left(n_{\lambda}^{M} \Delta_{0}^{M}+n_{\lambda}^{L} \Delta_{0}^{L}\right)} \\
& \times \sum_{k, l=0}^{\# M L-1} \iint d x_{k}^{\prime} d y_{l}^{\prime} e^{\frac{\pi i}{\lambda}\left(x_{k}^{\prime 2}+y_{l}^{\prime 2}\right)\left(\frac{1}{z}+\frac{1}{b_{L}}-\frac{1}{f_{L}}\right)} \cdot e^{\frac{-2 \pi i}{\lambda b_{L}}\left(\sigma^{\prime} x_{k}^{\prime}+\tau^{\prime} y_{l}^{\prime}\right)} \\
& \times \iint d u d v e^{\frac{\pi i}{\lambda}\left(u^{2}+v^{2}\right)\left(\frac{1}{z}+\frac{1}{a_{M}}-\frac{1}{f_{M}}\right)} \cdot e^{\frac{-2 \pi i}{\lambda z}\left(x_{k}^{\prime} u+y_{l}^{\prime} v\right)} \\
& \times \iint d \xi d \eta e^{\frac{2 \pi i}{\lambda a_{M}}\left(\xi^{2}+\eta^{2}\right)} \cdot e^{\frac{-2 \pi i}{\lambda a_{M}}(u \xi+v \eta)} \cdot U_{\lambda}^{O}(\xi, \eta) .
\end{aligned}
$$

Respectively,

$$
U_{\lambda}^{D}\left(P_{D}\right)=\iint d \xi d \eta h_{\lambda}\left(P_{O}, P_{D}\right) \cdot U_{\lambda}^{O}\left(P_{D}\right)
$$

with

$$
\begin{aligned}
h_{\lambda}\left(P_{O}, P_{D}\right)= & \frac{e^{\frac{2 \pi i}{\lambda}\left(a_{L}+z+b_{L}\right)}}{-i \lambda^{3} \cdot a_{M} \cdot z \cdot b_{L}} \cdot e^{\frac{2 \pi i}{\lambda}\left(n_{\lambda}^{M} \Delta_{0}^{M}+n_{\lambda}^{L} \Delta_{0}^{L}\right)} \cdot e^{\frac{\pi i}{\lambda b}\left(\sigma^{\prime 2}+\tau^{\prime 2}\right)} e^{\frac{\pi i}{\lambda a_{M}}\left(\xi^{2}+\eta^{2}\right)} \\
& \times \sum_{k, l=0}^{\# M L-1} \iint d x_{k}^{\prime} d y_{l}^{\prime} e^{\frac{\pi i}{\lambda}\left(x_{k}^{\prime 2}+y_{l}^{\prime 2}\right)\left(\frac{1}{z}+\frac{1}{b_{L}}-\frac{1}{f_{L}}\right)} \\
& \times \iint d u d v e^{\frac{\pi i}{\lambda}\left(u^{2}+v^{2}\right)\left(\frac{1}{z}+\frac{1}{a_{M}}-\frac{1}{f_{M}}\right)} \\
& \times \quad e^{\frac{-2 \pi i}{\lambda b_{L}}\left(\sigma^{\prime} x_{k}^{\prime}+\tau^{\prime} y_{l}^{\prime}\right)} \cdot e^{\frac{-2 \pi i}{\lambda z}\left(x_{k}^{\prime} u+y_{l}^{\prime} v\right)} \cdot e^{\frac{-2 \pi i}{\lambda a_{M}}(u \xi+v \eta)} .
\end{aligned}
$$

If the lens equations are fulfilled with $1 / f_{M}=1 / z+1 / a_{M}$ and $1 / f_{L}=1 / z+1 / b_{L}$, equation (25) can be simplified to

$$
\begin{aligned}
h_{\lambda}\left(P_{O}, P_{D}\right)= & \frac{e^{\frac{2 \pi i}{\lambda}\left(a_{L}+z+b_{L}\right)}}{-i \lambda^{3} \cdot a_{M} \cdot z \cdot b_{L}} \cdot e^{\frac{2 \pi i}{\lambda}\left(n_{\lambda}^{M} \Delta_{0}^{M}+n_{\lambda}^{L} \Delta_{0}^{L}\right)} \cdot e^{\frac{\pi i}{\lambda b_{L}}\left(\sigma^{\prime 2}+\tau^{\prime 2}\right)} e^{\frac{\pi i}{\lambda a_{M}}\left(\xi^{2}+\eta^{2}\right)} \\
& \times \sum_{k, l=0}^{\# M L-1} \iint d x_{k}^{\prime} d y_{l}^{\prime} e^{\frac{-2 \pi i}{\lambda b_{L}}\left(\sigma^{\prime} x_{k}^{\prime}+\tau^{\prime} y_{l}^{\prime}\right)} \iint d u d v e^{\frac{-2 \pi i}{\lambda z}\left(x_{k}^{\prime} u+y_{l}^{\prime} v\right)} \cdot e^{\frac{-2 \pi i}{\lambda a_{M}}(u \xi+v \eta)} .
\end{aligned}
$$

\subsection{Comparison between geometric and wave-optical approach}

Let there be a spot with $4 \mathrm{~mm}$ in diameter in a distance of $150 \mathrm{~mm}$ on-axis from the main lens plane. The light is captured by an array of $3 \times 3$ microlenses which cover $23 \times 23$ pixels each. The focal length of the main lens is set to $12.5 \mathrm{~mm}$, the focal length of the microlenses is $0.432 \mathrm{~mm}$, the f-number of the lenses is 3.8 . As mentioned before, in close-range imaging it is not always convenient to use the Fresnel approximation to describe the diffraction effects. Eq. (27) gives a quick way to check whether Fresnel is allowed or not, see [26]. This equation is based on a zeroing determination, checking the zeros of the diffraction integrand. It is

$$
F_{\# \min }=\frac{\gamma}{2 \cdot R_{n s t}}
$$

with

$$
R_{n s t}=\sqrt[4]{\frac{8(\alpha+\beta)-2 l}{\frac{1}{\alpha^{3}}+\frac{1}{\beta^{3}}}}
$$

as the bigger one of the first two zeros regarding real and imaginary part of the diffraction integrand. Furthermore it is $\gamma:=\frac{f}{\lambda}, \alpha:=\frac{a}{\lambda}, \beta:=\frac{b}{\lambda}$ and $R_{n s t}:=\frac{r_{n s t}}{\lambda}$. Here, (27) provides a minimal f-number for which Fresnel can be used without hesitation. Inserting the values for the main lens results in a minimal f-number of 7.3 to allow 
for Fresnel. However, (27) is rather pessimistic. Lower f-numbers might be tolerable as well, depending on what percentage of error is the personal pain barrier. It is helpful to check the percentage of error between Kirchhoff and Fresnel over a couple of f-numbers as in Figure 6. As can be seen, a f-number of at least 5.8 is needed to avoid a greater mistake of $10 \%$. Thus, Fresnel will not be sufficient to use. For the microlens, the equation provides a minimal f-number of 11.5. However, as can be seen in the right image of Figure 6 , the curve decreases faster than for the main lens. With the given f-number of 3.8 , a percentage error of only $2 \%$ is tolerable which hints that Fresnel would work for the latter part of the system. A combination of Kirchhoff and Fresnel is convenient.
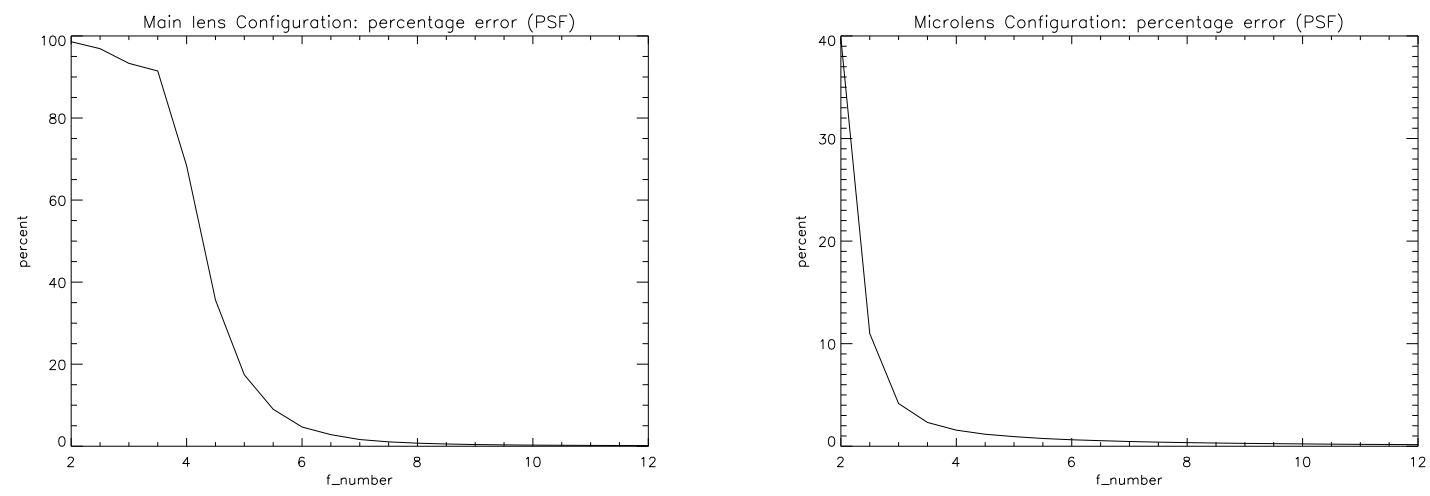

Figure 6: Percentage of error between the PSF by Kirchhoff and Fresnel. Left: main lens. Right: microlens.

The left image in Figure 7 depicts the image of the spot on the detector simulated by the geometric model, the middle and the right image show the effects of diffraction: first with a combination of Kirchhoff and Fresnel, second solely by Kirchhoff. As can be seen, the wave-optical approach generates a radial, symmetric PSF. The smearing effect is high, spreading the light intensity on not insignificant more pixels than the geometric approach. This might lead to artifacts in the images. However, the accuracy of the diffraction model is bought at a high price: While the geometric model provided the results in just a few seconds, the wave-optical approach with the diffraction formula by Kirchhoff took about 50 hours and 26 hours for the Kirchhoff-Fresnel combination. The geometric model might be a convenient solution for a quick simulation of the camera performance, but it should be kept in mind that the results are fraught with significant errors.
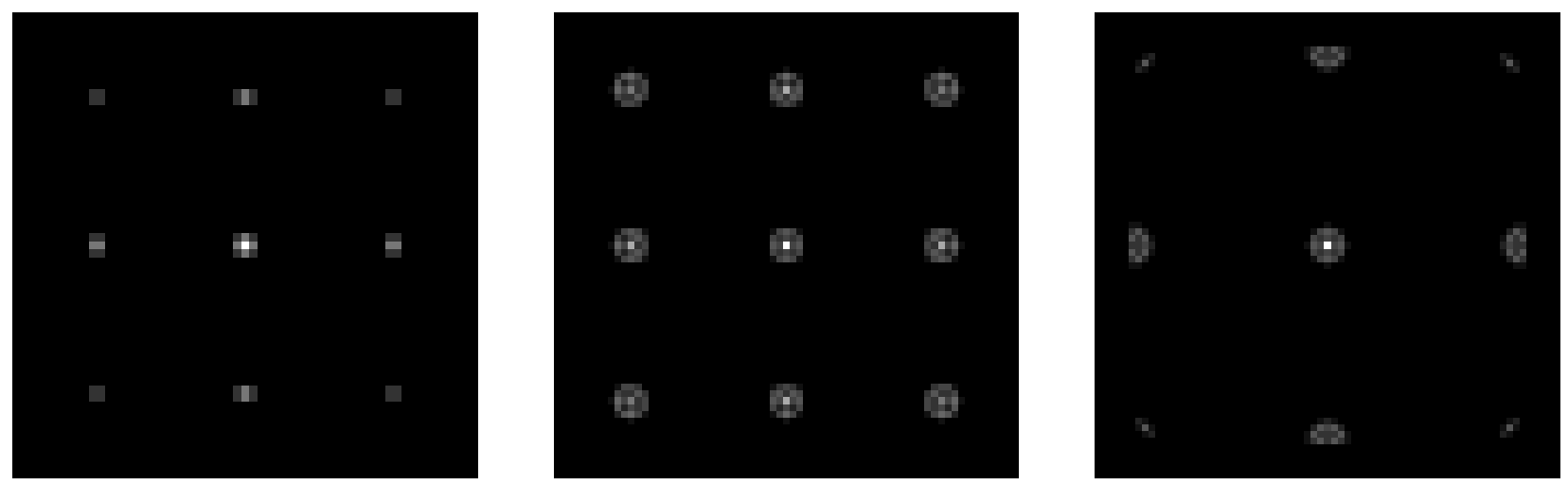

Figure 7: A light spot is captured by an array of $3 \times 3$ microlenses. Left: geometric model. Middle: wave-optical approach with Kirchhoff (main lens) and Fresnel (microlens). Right: wave-optical approach solely by Kirchhoff. 


\section{BRIEF COMPARISON OF A PLENOPTIC CAMERA AND A STEREO CAMERA SETUP FOR DEPTH ESTIMATION}

The computer-aided perception of the three dimensional environment is essential for a variety of applications in space missions (e.g. on-orbit servicing, rendezvous and docking maneuver). The modeling process relies on the calculation of a depth estimation from an ego-position to a distant object. While active means like laser scanners, radar and ultra sonic sounders inherently provide a depth value, camera based setups passively capture the objects within their field-of-view (FoV). The depth estimation is derived through triangulation from different viewpoints. Camera based approaches acquire the environment at a high geometrical and radiological resolution and at a much faster sample rate compared to other sensors (e.g. laser scanners) and have been used in several space exploration missions. Most commonly binocular camera setups were used in the past, that need to be re-calibrated in orbit after launch. Second, stereo configurations with relatively large baselines are difficult to maintain stable and possibly need frequent re-calibration, interrupting mission operations. Single-lens systems would inherently provide an advantage over stereo.

The following brief comparison of a standard stereo camera with a currently available plenoptic camera from Raytrix (R5) provides an indication of the usability of the plenoptic approach as an alternative to stereo configurations in space applications. Especially if the stereo baseline is kept short at $100 \mathrm{~mm}$, which means that a high calibration stability of the exterior orientation is achievable due to high thermo-mechanical rigidness. First, a close-range application is assumed at distances between 0 and $1 \mathrm{~m}$ and a mid-range scenario between 1 and $10 \mathrm{~m}$. The parameters for both are as follow (see Table 1 below). The uncertainty of the depth estimation was modeled with $1 / 10$ sub-pixel accuracy. For the stereo setup, the calibration uncertainty was modeled with less than $0,2^{\circ}$ degrees for exterior rotation.

\begin{tabular}{lcl} 
parameter & value & units \\
\hline pixel size & 10 & $\mu m$ \\
sensor dim & $1024 \times 1024$ & pixel \\
focal length & 30,5 & $\mathrm{~mm}$ \\
base length & 100 & $\mathrm{~mm}$ \\
FOV & 65,24 & degree
\end{tabular}

(a) Industrial camera

\begin{tabular}{lcl} 
parameter & value & units \\
\hline pixel size & 5,5 & $\mu m$ \\
sensor dim & $2048 \times 2048$ & pixel \\
focal length (main) & 12,5 & $m m$ \\
FOV & 65,24 & degree
\end{tabular}

(b) Raytrix R5

Table 1: Parameters of the stereo setup and Raytrix R5 plenoptic camera

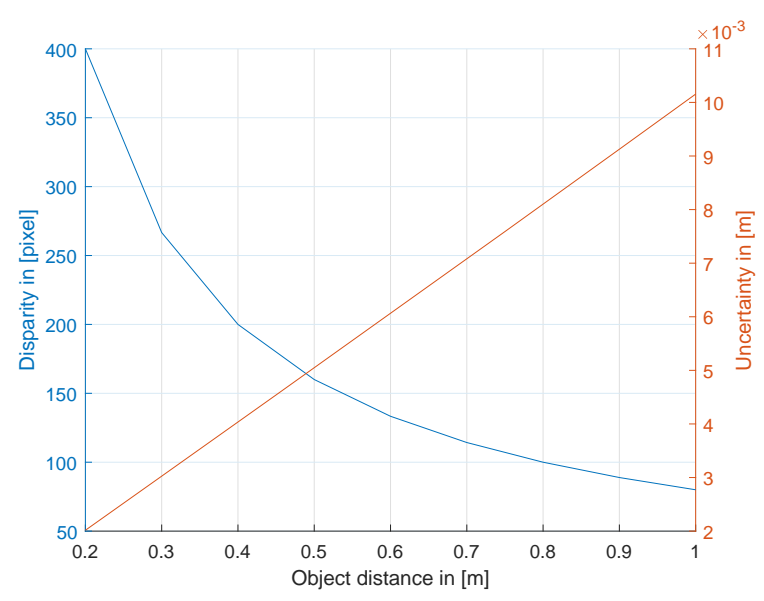

(a) Stereo setup

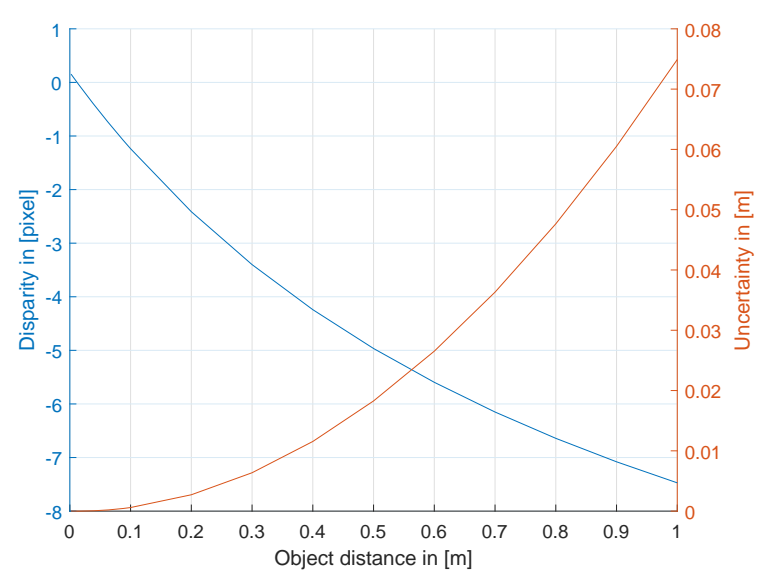

(b) Plenoptic R5

Figure 8: Depth discriminating power and uncertainty of the distance measurement as a function of object distance with in a range of 0 to $1 m$

Within the range of 0 to 1 meter, the stereo depth measurement is only feasible beginning at $\approx 0.5 \mathrm{~m}$ (assuming $80 \%$ overlap). From there, the measurement uncertainty is lower compared to the plenoptic camera. 
Based on the above configuration the plenoptic camera is suited in the range less than $\approx 1 \mathrm{~m}$. Beyond that range, the estimation uncertainty is judged to high. Its range is overall limited to $7 \mathrm{~m}$.

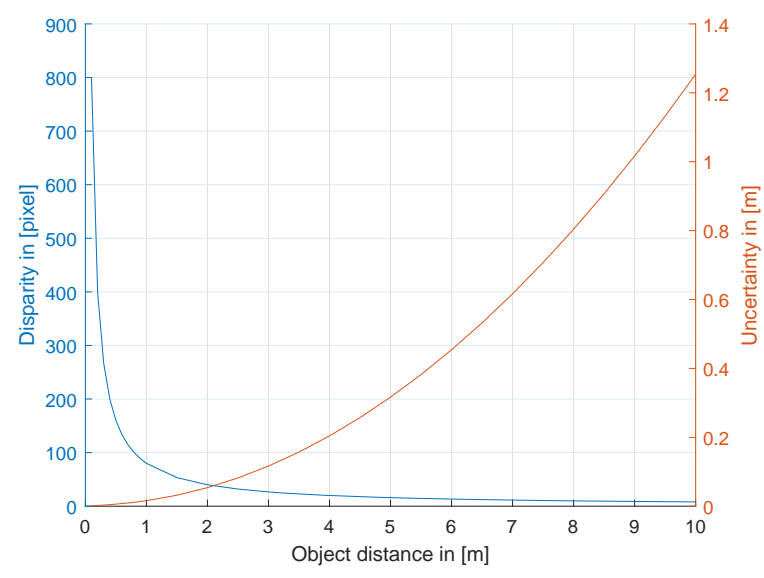

(a) Stereo setup

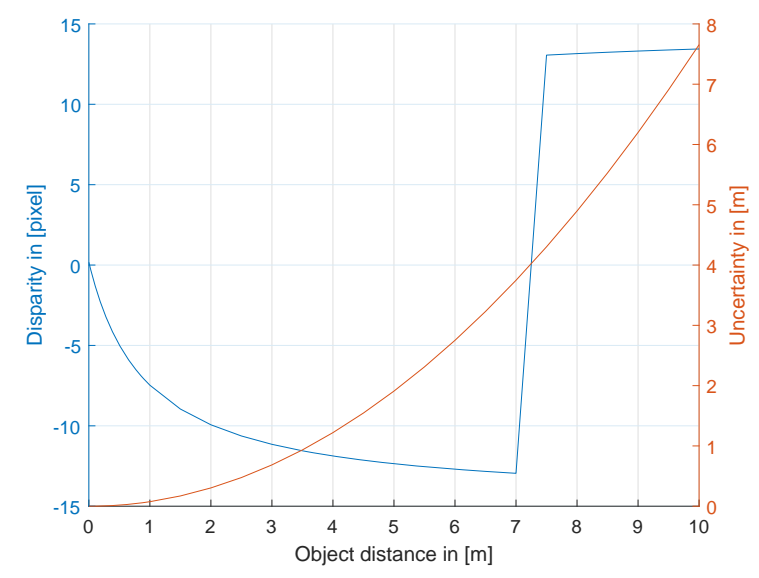

(b) Plenoptic R5

Figure 9: Depth discriminating power and uncertainty of the distance measurement as a function of object distance with in a range of 1 to $10 \mathrm{~m}$

\section{SUMMARY AND OUTLOOK}

This work discusses the potential advantages of plenoptic cameras for close range imaging during in-situ planetary exploration. The ability of multi-focus plenoptic cameras to record scenes with an extended depth of field without the need for refocusing makes them an interesting concept for future hand lens imagers. This gives the possibility to avoid the use of a complex focus mechanism for the camera while at the same time providing the same or even more data products. As a first step towards plenoptic hand lens imagers, we outlined the mathematical description of both, a geometric and a wave-optical model of a plenoptic camera. While the geometric approach gives a good and quick impression of plenoptic imaging by a microlens array, all effects of diffraction are neglected. In some cases, due to high calculation times, it is convenient to work with the geometric model in order to investigate basic phenomena. However, in close-range it would be wise not to dispense with the diffraction effects as can be seen in the results gained with the wave-optical model. In contrast to the geometric model, the wave-optical model shows, that diffraction leads to light falling on adjacent pixels, which needs to be considered in the design of light field processing algorithms.

Thus in the future, a hybrid formulation of the geometric model combined with wave-optical methods, used in the geometrical theory of diffraction or the so-called boundary-diffracted wave theory, might be a solution to overcome the otherwise large computing time of the wave-optical model. Hence, this might be a way to combine the advantage of both models, short computing time on the one hand and physical correctness on the other hand.

In addition, a preliminary comparison between the plenoptic camera and a conventional stereo camera system suggests that the light field camera can be a good substitute for such a system. A more detailed comparison between those camera systems is ongoing work.

\section{REFERENCES}

[1] Ng, R., Levoy, M., Bredif, M., Duval, G., Horowitz, M., and Hanrahan, P., [Light field photography with a hand-held plenoptic camera], Technical Report CSTR 2005-02, Stanford Univ (April 2005).

[2] Perwass, C. and Wietzke, L., "Single lens 3d-camera with extended depth-of-field," Proc.SPIE 8291, 8291 - 8291 - 15 (2012).

[3] Stoykova, E. and Sainov, V., "Institute of optical materials and technologies." [Online].Available http: //www.clf.bas.bg/page_06/Integral_Imaging.pdf. (Accessed: 6 May 2014). 
[4] Lam, E. Y., "Computational photography with plenoptic camera and light field capture: tutorial," J. Opt. Soc. Am. A 32, 2021-2032 (Nov 2015).

[5] Georgiev, T. and Lumsdaine, A., "Depth of Field in Plenoptic Cameras," in [Eurographics 2009 - Short Papers], Alliez, P. and Magnor, M., eds., The Eurographics Association (2009).

[6] Fiss, J., Curless, B., and Szeliski, R., "Refocusing plenoptic images using depth-adaptive splatting," In: $\operatorname{ICCP}(2014)$.

[7] Hansen, M. and Holk, E., "Depth map estimation for plenoptic images," (2011).

[8] Lippmann, G., "Epreuves reversibles donnant la sensation du relief," Journal of Theoretical and Applied Physics 7, pp. 821-825 (1908).

[9] Adelson, E. H. and Bergen, J. R., "The plenoptic function and the elements of early vision," Computational Models of Visual Processing, pp. 3-20 (1991).

[10] Levoy, M. and Hanrahan, P., "Light field rendering," in SIGGRAPH '96 (1996).

[11] Georgiev, T. G. and Lumsdaine, A., "Focused plenoptic camera and rendering," Journal of Electronic Imaging vol. 19, Issue 2, pp. 28 (Mar. 3, 2010).

[12] Georgiev, T. and Lumsdaine, A., "The multifocus plenoptic camera," in [ISEST/SPIE Electronic Imaging], 829908-829908, International Society for Optics and Photonics (2012).

[13] Edgett, K. S., Caplinger, M. A., Maki, J. N., vine, M. A. R., Ghaemi, F. T., McNair, S., Herkenhoff, K. E., Duston, B. M., Willson, R. G., Yingst, R. A., Kennedy, M. R., Minitti, M. E., Sengstacken, A. J., Supulver, K. D., Lipkaman, L. J., Krezoski, G. M., McBride, M. J., Jones, T. L., Nixon, B. E., Beek, J. K. V., Krysak, D. J., , and Kirk, R. L., "Curiosity's robotic arm-mounted Mars Hand Lens Imager (MAHLI): Characterization and calibration status, MSL MAHLI Technical Report," tech. rep., Malin Space Science Systems (2015).

[14] Edgett, K., Ravine, M., Caplinger, M., Ghaemi, F., Schaffner, J., Malin, M., Baker, J., Dibiase, D., Laramee, J., Maki, J., Willson, R., Bell, J., Cameron, J., Dietrich, W., Edwards, L., Hallet, B., Herkenhoff, K., Heydari, E., Kah, L., Lemmon, M., Minitti, M., Olson, T., Parker, T., Rowland, S., Schieber, J., Sullivan, R., Sumner, D., Thomas, P., and Yingst, R., "The Mars Science Laboratory (MSL) Mars Hand Lens Imager (MAHLI) Flight Instrument," in [Lunar and Planetary Science Conference], Lunar and Planetary Inst. Technical Report 40, 1197 (Mar. 2009).

[15] Yingst, R. A., Edgett, K. S., Kennedy, M. R., Krezoski, G. M., McBride, M. J., Minitti, M. E., Ravine, M. A., and Williams, R. M. E., "MAHLI on Mars: lessons learned operating a geoscience camera on a landed payload robotic arm," Geoscientific Instrumentation, Methods and Data Systems 5, 205-217 (June 2016).

[16] Ghaemi, F. T., "Design and fabrication of lenses for the color science cameras aboard the Mars Science Laboratory rover," Optical Engineering 48(10), 103002-103002-15 (2009).

[17] DiBiase, D. R. and Laramee, J., "Mars hand lens imager: Lens mechanical design," in [2009 IEEE Aerospace conference], 1-10 (Mar. 2009).

[18] Wetzstein, G., Ihrke, I., Lanman, D., and Heidrich, W., "Computational Plenoptic Imaging," Computer Graphics Forum 30(8), 2397-2426 (2011).

[19] Ng, R., [Digital light field photography,], Ph.D. thesis, Stanford University, Stanford, CA, USA (2006).

[20] Krutz, U., [Analytische Betrachtung optisch abbildender Sensorsysteme im Rahmen der AsteroidFinderMission], Optimedien Verlag e.K., Oldenburg (17. June 2011).

[21] Jahn, H. and Reulke, R., [Systemtheoretische Grundlagen optoelektronischer Sensoren], Akademie Verlag GmbH, Berlin (1995).

[22] Hecht, E., [Optik], Oldenbourg Wissenschaftsverlag, ISBN: 3486588613 (8. April 2009).

[23] Mendlovic, D., Zalevsky, Z., and Konforti, N., "Computation considerations and fast algorithms for calculating the diffraction integral," Journal of Modern Optics 44(2), 407-414 (1997).

[24] Xin Jin, Li Liu, Y. C. and Dai, Q., "Point spread function and depth-invariant focal sweep point spread function for plenoptic camera 2.0," Optics Express 25, No 9 (1. May 2017).

[25] Turola, M., [Investigation of plenoptic imaging systems: a wave optics approach], Unpublished Doctoral thesis, City University London (2016). 
[26] Talbiersky, U., "A new method to validate the usage of fresnel approximation instead of kirchhoff diffraction formula for calculations concerning camera systems," Proc.SPIE 7717, 7717 - 7717 - 11 (2010).

[27] Jahns, J., [Photonik: Grundlagen, Komponenten und Systeme], Oldenbourg Wissenschaftsverlag, ISBN 9783486254259 (2001). 